faibles que $1 \%$ renferment beaucoup plus d'amidon (révélateur de la margarine) que les beurres naturels authentiques.

4. Il convenait donc de modifier la méthode officielle et d'aboutir à une technique suffisamment "quantitative" pour distinguer avee certitude certains beurres renfermant naturellement de l'amidon, des beurres additionnés de faibles quantités de margarine.

5. Une méthode a été mise au point et décrite en détail qui permet d'aboutir à un culot de centrifugation renfermant, à l'état à peu près pur, la totalité des amidons de la prise d'essai.

Le culot de centrifugation ainsi obtenu peut être :

Soit soumis à une numération à l'hématimètre (si l'on compte moins de 100.000 grains d'amidon dans le culot c'est qu'il n'y avait pas de margarine dans le beurre ou seulement moins de $1 \%$; les beurres naturels ne renferment, dans les mêmes conditions, que quelques milliers de grains d'amidon - ce qui lève toute équivoque) ;

Soit transformé en empois de $10 \mathrm{~cm}^{3}$ et coloré à l'iode (si l'on ne constate pas de coloration, e'est qu'il n'y avait pas de margarine ou seulement moins de $0,1 \%$; les beurres naturels renfermant de l'amidon ne donnent, dans les mêmes conditions, aueune coloration).

\title{
ACTION DE QUELQUES ANTIBIOTIQUES SUR LES FERMENTS DU FROMAGE ET LA FLORE MICROBIENNE PATHOGENE DE LA MAMELLE (1) \\ par
}

le Pr S. OVEJERO, le Dr M. DIEZ et la Doctoresse R. PASCUAL

L'existence d'infections dans la mamelle des animaux laitiers crée un important problème qui affecte autant la quantité que la qualité du lait.

Les mammites bactériennes, en occasionnant des perturbations profondes dans le fonctionnement du pis, diminuent la quantité de lait et altèrent la composition biochimique de ce complexe biologique de si grande importance dans l'alimentation de l'homme et dans l'élevage et exploitation de certaines espèces animales.

Nocard et Mollereau décrivent pour la première fois en 1894 le processus clinique qui, se présentant sous une forme voilée chez les bovins laitiers, va se transmettant de ces animaux, en apparence sains, à ceux de leur voisinage, créant un foyer dont le développement produit de légères altérations dans la mamelle et le lait.

(1) Travail patronné par le Comité National Laitier Espagnol. 
et donne lieu aux mammites de conséquences économiques si graves.

Depuis les premiers travaux des investigateurs français cités plus haut jusqu'à nos jours, les études se sont poursuivies dans tous les pays touchant ce processus infecto-contagieux dont les formes sub-cliniques sous lesquelles il se présente, c'est-à-dire avec des signes externes peu manifestes, contribuent à sa diffusion entre la population bovine laitière, créant ainsi dans les étables un grand nombre de malades en état latent, et causant, en conséquence, des pertes économiques d'importance diverse dues à des produits laitiers de qualité inférieure ou à un pauvre approvisionnement en lait des centres urbains.

Des recherches effectuées à Weybridge par STABLEForth et Collaborateurs établissent que $59 \%$ des mammites sont produites par des Streptococcus des types agalactiae, dysgalactiae, uberis et pyogenes.

Nos travaux, réalisés sur des bovins hollandais formant un noyau d'étables situées dans le périmètre d'une grande ville, nous ont conduits aux résultats suivants :

$\begin{array}{ll}\text { Mammites streptocociques } \ldots \ldots \ldots \ldots \ldots \ldots \ldots \ldots \ldots \ldots \ldots \ldots \ldots \ldots & 87 \% \\ \text { Mammites staphylocociques } \ldots \ldots \ldots \ldots \ldots \ldots & 13 \%\end{array}$

avec pourcentages, pour les premières, de

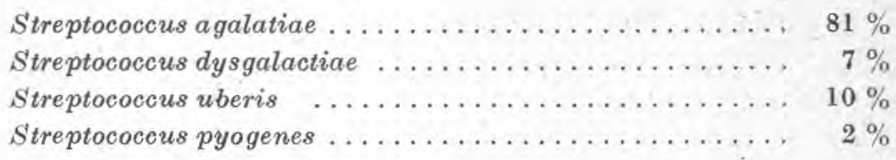

Outre les différents Streptococcus producteurs de mammites, il existe une autre flore bactérienne pathogène qui, se logeant également pendant des périodes plus ou moins longues dans la mamelle des femelles laitières, provoque des troubles dans la sécrétion et des altérations dans le lait et les produits dérivés.

Dans le but d'évaluer la gravité de cette maladie qui, dû à l'aspect normal des animaux atteints, passe souvent inaperȩue par l'éleveur, il sera intéressant de connaître les résultats suivants obtenus par les investigateurs anglais : $8 \%$ seulement des vaches contaminées par le Streptococcus agalactiae présentaient des caractères anormaux dans leur lait.

SHAw et BEAm ont pu observer, dans une comparaison de la production laitière de 36 vaches, à mamelles saines les unes, et malades les autres, que la diminution du rendement en lait chez les animaux infectés est d'environ $21 \%$, atteignant celle de la matière grasse jusqu'à $25 \%$. 
Notre illustre ami, le Pr Seelemann, de Kiel, en insistant sur l'importance de l'absence d'altérations dans la mamelle de vaches atteintes, affirme que \& souvent une glande mammaire peut contenir des Streptococcus et produire une sécrétion anormale, alors qu'il n'apparaît dans son tissu aucun changement susceptible d'être apprécié par l'examen clinique n.

L'étude systématique de plus de mille bovins laitiers, au moyen des épreuves de laboratoire, nous a confirmé la grande fréquenee de laits bactériologiquement anormaux provenant de mamelles cliniquement saines. De là l'intérêt extraordinaire que doivent avoir les industries laitières à contribuer à la lutte contre les infections du pis.

Dans un essai réalisé par MinetT et Martin sur des vaches Ayrshires et Hollandaises, et au cours duquel ils ont tenu compte de tous les facteurs individuels qui peuvent influer sur la production du lait, ils arrivent à évaluer les pertes totales de lait dues à la diminution de la sécrétion à $10,8 \%$ pour la race Ayrshire et à $19,5 \%$ pour le bétail bovin hollandais soumis au contrôle. Ces pertes se rapportent à des animaux dont la maladie se présente sous une forme latente.

Bien connues sont les altérations observées dans la composition du lait provenant de vaches attaquées de mammite, qui oceasionnent, entre autres, des perturbations sérieuses dans l'industrie fromagère, vu que, sous une apparence normale, le lait renferme moins de caséine et de matière grasse, ce qui, joint à une augmentation évidente du chlorure de sodium et à des modifications dans la réaction et la structure physico-chimique, produit une matière première peu apte à l'obtention de fromages de qualité ou de produits dérivés devant subir une fermentation lactique.

Le fait de considérer l'importance économique des mammites bactériennes sous l'angle de l'économie laitière mondiale, nous permet d'accepter l'opinion du Pr KästLI dans sa recommandation à l'Office International des Epizooties (1948), que "La prophylaxie de cette épizootie d'évolution chronique doit commencer par un contrôle régulier de l'état sanitaire des mamelles et par l'examen bactériologique du lait présentant des altérations pathologiques ".

En résumé des considérations antérieures, nous pouvons souligner la nécessité de lutter contre les mammites du bétail laitier, en utilisant les méthodes diagnostiques appropriées, ainsi qu'une thérapeutique capable d'assurer la disparition de la flore microbienne pathogène de la mamelle.

Comme médications les plus modernes et efficaces contre la mammite, on a employé les sulfamides et les antibiotiques. Sans nous 
arrêter à l'étude de ces remèdes, nous pouvons toutefois rappeler qu'entre les antibiotiques, les suivants ont été appliqués dans les pis contaminés par les différentes bactéries: Pénicilline, Streptomycine, Auréomycine, Chloromycétine, Subtiline, Bacitracine, Terramycine, et autres de moindre importance.

L'efficacité de la Pénicilline sur les Streptococcus et Staphylococcus des mammites, qui en fait conseiller l'emploi sur une grande échelle, crée, d'autre part, un problème dû à l'introduction, dans le lait, d'une substance agissant à la fois sur les bactéries pathogènes et sur la flore microbienne productrice d'acide lactique qui régit les fermentations nécessaires dans l'élaboration des dérivés du lait.

Les excipient aqueux ou les différentes graisses végétales, minérales ou animales utilisés pour l'application de la Pénicilline intramammaire, ainsi que l'association de substances amélioratrices de l'action thérapeutique de cet antibiotique, telles que le monostéarate d'aluminium ou la Procaïne (Novocaïne), ont une influence remarquable sur l'élimination des antibiotiques.

La Station des Recherches Laitières de Hillerd a communiqué les résultats suivants concernant 12 vaches : La concentration de Pénicilline dans le lait variait, après douze heures, de 5,3 à 19,6 U.I. par centimètre cube, descendant à 0,11 U.I. et à 1,4 U.I. par centimètre cube au bout de vingt-quatre heures.

Nous pouvons, en général, corroborer l'opinion du $\mathrm{P}^{\mathrm{r}} \mathrm{K}$. E. Tноме́ lorsqu'il affirme, d'accord avec de nombreux investigateurs (Schalm et Canelberry, Stevenson, Kelly et Bell, Käastit, Seflemañ, etc.), que la quantité de Pénicilline contenue dans le lait de vaches soumises à traitement, est élevée passé douze heures, diminue après vingt-quatre heures, apparaissant au bout de trente-six heures, et après la troisième traite, virtuellement exempt de Pénicilline.

La période de trente-six heures, considérée normale pour l'élimination de l'antibiotique par le lait, se montre insuffisante lorsque, par des substances retardatrices favorisant l'action eurative, le contact de la Pénicilline et du liquide mammaire infecté se trouve prolongé.

Les travaux de JEPSEN, en coïneidence avec les recherches effectuées par SEELEMANN et Collaborateurs, confirment qu'après emploi de la Pénicilline-Procaïne intramammaire, le lait ne contient passé soixante-douze heures, que des traces de ce médicament biologique.

L'action inhibitive de la croissance bactérienne, découverte par Fleming sur les lames de Staphylococcus, et étudiée par la suite sur un grand nombre d'espèces bactériennes (Fleming, Florex 
et Chain, 1941), atteint également la flore microbienne de l'acide lactique, ainsi que la vaste gamme des germes qui interviennent dans les fermentations des différentes espèces de fromages.

Les altérations produites par la Pénicilline sur la flore bactérienne Gram-positive, sans modifier sensiblement les bactéries Gramnégatives, ainsi que sa répercussion possible sur la population enfantine par la création d'un état d'hypersensibilité (BRYAN), conseillent la prise de mesures tendant à contrôler l'emploi de ce médicament d'utilité bien reconnue, mais qui offre aussi des dangers évidents pour la qualité des produits dérivés du lait.

L'usage abusif de la Pénicilline dans le traitement des mammites provoque, non seulement les altérations de la flore de l'acide lactique si nécessaire dans les processus de maturation, sinon encore la sélection des germes pathogènes les plus résistants, maintenant ainsi un foyer d'infection latente, source de presque toutes les réapparitions de mammites à l'étable.

J. JACQUet et M. JuLienne ont constaté dans le Calvados (France) la disparition des Streptococcus et l'apparition des Staphylococcus pathogènes chez les bovins traités à la Pénicilline.

D'une part, J. JACQuet, R. Richou, Mc Steeg, C. Gerbaux et P. JULienne, dans un essai effectué sur une grande échelle durant les années 1947 à 1951 , observent ehez les animaux traités à la Pénicilline la diminution des Streptococcus agalactiae de 24 à $2 \%$ et l'accroissement des Staphylococcus de 9 à $22 \%$, ainsi que celui des bacilles Coli de 1 à $5 \%$. Cette possibilité de création d'une flore mierobienne résistante aux antibiotiques, jointe à la nécessité d'éviter, dans les industries, l'utilisation de laits contenant de la Pénicilline, incitent à solliciter que les Vétérinaires soient les seuls capables d'utiliser ees modernes remèdes des mammites, empêchant ainsi l'usage immodéré de ces actifs composés biochimiques et contrôlant, en outre, la destination donnée au lait provenant de vaches soumises à traitement.

De notre part, nous avons réalisé des essais expérimentaux de fabrication de fromages avec du lait de vache, sur la base d'une technique de coagulation en deux heures et demie, à l'objet d'obtenir un type de fromage "demi-dur léonais" (de la province espagnole de Léon).

Avec l'emploi de laits contenant des quantités eroissantes de Pénicilline nous avons obtenu les résultats suivants :

\section{a) Fromages sans pénicilline}

Fromages d'un mois : Bon aspect; bonne consistance ; onctueux ; goût agréable. 
Fromages de deux mois : Bon aspect; consistance plus grande ; yeux abondants; goût agréable.

Fromages de deux mois et demi : Bon aspect; consistance durcie ; yeux abondants; goût agréable; bonne odeur.

\section{b) Fromages avec pénicilline}

Fromages avec 0,25 U. I. par centimètre cube: Bon aspect; bonne consistance; onctueux ou durci suivant l'âge; quantité d'yeux suivant l'âge ; goût agréable.

Fromages avec 0,50 U. I. par centimètre cubes : Mêmes caractères organoleptiques que les précédents,

Fromages avec 1 U. I. par centimètre cube : Aspect médiocre; fortement durcis ; goût amer.

Fromages avec 2 U. I. par centimètre cube: Aspect médiocre ; très durs; goût très amer; sans odeur.

Fromages avec 5 U. I. par centimètre cube: Caractères similaires à ceux de 2 U. I.

Fromages avec $10 \mathrm{U} I$. par centimètre cube : Caractères similaires à ceux de 2 U. I.

De cette épreuve, nous pouvons déduire que des quantités de 0,25 U. I. à 0,50 U. I. de Pénicilline par centimètre cube de lait n'influencent pas la fermentation de l'espèce de fromage élaboré. Des concentrations supérieures de 1, 2, 5, 10 U. I. du médicament, altèrent la flore de fermentation et, conséquemment, la qualité de ce type de formage espagnol.

Les souches des différents ferments isolés de fromages fabriqués avec du lait contenant de 1 à 10 U. I. de Pénicilline par centimètre cube ont subi un changement dans leurs caractères biologiques, ayant été observé au laboratoire que leur développement dans les milieux de culture utilisés (milieux à base de lactosérum) est lent et la coloration irrégulière, choses toutes révélatrices d'altérations dans leur métabolisme et dans la composition biochimique du soma bactérium.

Les réensemencements successifs dans des milieux appropriés permettent la récupération des caractères normaux de ces germes qui ont subi une action partielle de l'antibiotique élaboré par le Penicillum notatum Westling ou le P. chrysogenum Thom.

A l'objet de connaître le processus de fermentation du type de fromage fabriqué, nous avons effectué, durant les diverses périodes de sa phase d'acidification et de maturation, les recherches bactériologiques opportunes, des suites desquelles nous avons identifié les ferments lactiques suivants : 
Streptococcus cremoris (ORLA-JENSEN) ;

Streptococcus lactis (LISTER, LoHisis;

Streptobacterium plantarum (ORLA JENSEN).

Streptobacterium casei (ORLA JENSEN) ;

Microbacterium lactis (ORLA JENSEN).

Une fois la flore de fermentation du fromage " demi-dur léonais » connue, nous avons réalisé une étude de la sensibilité de ces espèces bactériennes, en utilisant le test des disques avec différents antibiotiques préparés par Difco Laboratories, Michigan, U.S.A.

Les résultats obtenus avec des concentrations variées d'antibiotiques divers, et en employant des milieux de culture appropriés, à base de lactosérum, sont mentionnés dans les tableaux ci-après :

TABLEAU I

DIAMËTRE EN MM. DES ZONES D'INHIBITION DE LA GROISSANGE AVEG DISQUES DE $6,5 \mathrm{MM} / \mathrm{D}$

\begin{tabular}{|c|c|c|c|c|c|c|c|c|c|}
\hline \multirow[b]{2}{*}{ Germe } & \multicolumn{3}{|c|}{ Pénieilline } & \multicolumn{3}{|c|}{ Streptomycine } & \multicolumn{3}{|c|}{ Auréomyeine } \\
\hline & $\begin{array}{r}10 \\
\text { U. I. }\end{array}$ & $\stackrel{5}{\mathrm{U} . \mathrm{I}}$ & $\begin{array}{r}0,5 \\
\text { U. I. }\end{array}$ & $\begin{array}{l}100 \\
\text { mog. }\end{array}$ & $\begin{array}{c}10 \\
\text { meg. }\end{array}$ & $\begin{array}{c}1 \\
\operatorname{meg} .\end{array}$ & $\begin{array}{r}60 \\
\operatorname{meg} .\end{array}$ & $\begin{array}{c}30 \\
\text { meg. }\end{array}$ & $\begin{array}{r}10 \\
\operatorname{meg} .\end{array}$ \\
\hline $\begin{array}{r}\text { Streptococcus cre- } \\
\text { moris ....... }\end{array}$ & 13 & 1 & 10 & 23 & 13 & 11.5 & 26 & 23 & 22 \\
\hline Streptococcus lac. & & & & & & & & & \\
\hline $\begin{array}{c}\text { tis } \ldots \ldots \ldots \\
\text { Streptobacterium }\end{array}$ & 14 & 12 & 11 & 22 & 17,5 & 12 & 17 & 15,5 & 14 \\
\hline $\begin{array}{r}\text { plantarum.... } \\
\text { Streptobacterium }\end{array}$ & 16,5 & 14 & 11,5 & 19 & 14 & 12 & 16 & 16 & 16 \\
\hline casei $\ldots \ldots$ & 22 & 17,5 & 8,5 & 35 & 23 & 17 & 35 & 32 & 27 \\
\hline Microbacterium & & & & & & & & & \\
\hline lactis ...... & 11 & 9,5 & 8,5 & 24 & 14 & 11 & 17 & 16 & 15,5 \\
\hline
\end{tabular}

La faible action de la Bacitracine sur les ferments utiles conseille de profiter de ce caractère qui coïncide avec sa forte influence inhibitrice sur la flore pathogène de la mammite (tableaux I, II, III, IV).

De l'étude de ces six antibiotiques, face à la flore microbienne pathogène, la plus fréquente dans les mammites bovines, et face aussi aux ferments lactiques isolés du type de fromage élaboré dans la région léonaise (Espagne), nous estimons d'intérêt l'évaluation, moyennant une ample expérimentation, de l'utilité de la Bacitracine dans le traitement des affections mammaires, vu qu'il est possible que cet antibiotique constitue un bon remède contre les infections 
TABLEAU II

DIAMÉTRE EN MM. DES ZONES D'INHIBITION DE LA CROISSANGE AVEG DISQUES DE $5,5 \mathrm{MM} / \mathrm{D}$

\begin{tabular}{|c|c|c|c|c|c|c|c|c|c|}
\hline \multirow[b]{2}{*}{ Germe } & \multicolumn{3}{|c|}{ Bacitracine } & \multicolumn{3}{|c|}{ Chloromycétine } & \multicolumn{3}{|c|}{ Terramycine } \\
\hline & $\begin{array}{l}20 \\
\mathrm{U} .\end{array}$ & $\begin{array}{l}10 \\
\text { U. }\end{array}$ & $\begin{array}{l}2 \\
\mathrm{U}\end{array}$ & $\begin{array}{c}60 \\
\text { meg. }\end{array}$ & $\begin{array}{c}30 \\
\text { meg. }\end{array}$ & $\begin{array}{r}10 \\
\text { meg. }\end{array}$ & $\begin{array}{c}60 \\
\text { meg. }\end{array}$ & $\begin{array}{c}30 \\
\mathrm{meg} .\end{array}$ & $\begin{array}{c}10 \\
\mathrm{meg} .\end{array}$ \\
\hline $\begin{array}{r}\text { Streptococcus ore- } \\
\text { moris } \ldots \ldots \ldots\end{array}$ & 15 & 10.5 & 8.5 & 90 & 20 & 16.5 & 18 & 16,5 & 15 \\
\hline $\begin{array}{r}\text { Streptococeus lac- } \\
\text { tis ........ }\end{array}$ & 9 & - & - & 24 & 24 & 18 & 17 & 16 & 14 \\
\hline Streptobacterium & & & & & & & & & \\
\hline $\begin{array}{l}\text { plantarum } \\
\text { Streptobacterium }\end{array}$ & - & - & - & 22 & 17,5 & 13 & 18 & 16 & 15 \\
\hline $\begin{array}{c}\text { casei } \ldots \ldots \ldots \\
\text { Microbacterium }\end{array}$ & 32 & 30 & 21,5 & 36 & 20 & 16 & 35 & 35 & 23 \\
\hline lactis $\ldots \ldots$. & 12 & - & - & 23 & 18,5 & 16 & 17 & 16 & 12,5 \\
\hline
\end{tabular}

TABLEAU III

DLAMĖTRE EN MM. DES ZONES D'INHIBITION DE LA CROISSANGE AVEC DISQUES DE $6,5 \mathrm{MM} / \mathrm{D}$

\begin{tabular}{|c|c|c|c|c|c|c|c|c|c|}
\hline \multirow[b]{2}{*}{ Germe } & \multicolumn{3}{|c|}{ Pénicilline } & \multicolumn{3}{|c|}{ Streptomycine } & \multicolumn{3}{|c|}{ Auréomycine } \\
\hline & $\begin{array}{l}10 \\
\text { U. I. }\end{array}$ & $\begin{array}{l}5 \\
\text { U. I. }\end{array}$ & $\begin{array}{l}0,5 \\
\text { U. I. }\end{array}$ & $\begin{array}{l}100 \\
\text { meg. }\end{array}$ & $\begin{array}{c}10 \\
\text { meg. }\end{array}$ & $\begin{array}{c}\text { I } \\
\text { meg. }\end{array}$ & $\begin{array}{c}60 \\
\text { meg. }\end{array}$ & $\begin{array}{c}30 \\
\mathrm{meg} .\end{array}$ & $\begin{array}{c}10 \\
\text { meg. }\end{array}$ \\
\hline Streptococcus aga. & & & & & & & & & \\
\hline $\begin{array}{r}\text { lactiae } \ldots . . . \\
\text { Streptococcus aga. }\end{array}$ & 28 & 21 & 16 & 20 & 17 & 13,5 & 23,5 & 20 & 17 \\
\hline $\begin{array}{r}\text { lactiae } \ldots \ldots \\
\text { Streptococeus aga. }\end{array}$ & 31 & 24 & 17,5 & 13 & 11,5 & 9 & 24 & 19,5 & 16 . \\
\hline $\begin{array}{r}\text { lactiae ...... } \\
\text { Streptococcus dys. }\end{array}$ & 26,5 & 18 & 14,5 & 13,5 & 10,5 & 8,5 & 26 & 21 & 19 \\
\hline $\begin{array}{c}\text { Streptococcus dys- } \\
\text { galactiae } \ldots .\end{array}$ & 21 & 16,5 & 11 & 13 & 10 & 8 & 16 & 11 & 9,5 \\
\hline $\begin{array}{r}\text { Streptococous ube } \\
\quad \text { ris } \ldots \ldots \ldots\end{array}$ & 24 & 19 & 15,5 & 12 & 12 & 9,5 & 26,5 & 20 & 17 \\
\hline Staphilococcus .. & 23 & 22 & 13 & 19 & 16,5 & 10 & 23,5 & 16 & 11 \\
\hline
\end{tabular}

du pis, sans altérer profondément la flore lactique nécessaire dans les processus de maturation de certains types de fromages.

La Bacitracine fut découverte l'année 1945 par JoHnson, Anker et Meleney, à partir de la flore microbienne de blessures infectées souffertes par des malades hospitalisés au "Presbyterian 
TABLEAU IV

DIAMETTRE EN MM. DES ZONES D'INHIBITION DE LA CROISSANCE AVEC DISQUES DE $6,5 \mathrm{MM} / \mathrm{D}$

\begin{tabular}{|c|c|c|c|c|c|c|c|c|c|}
\hline \multirow{2}{*}{ Germe } & \multicolumn{3}{|c|}{ Bacitracine } & \multicolumn{3}{|c|}{ Chloromyeétine } & \multicolumn{3}{|c|}{ Terramycine } \\
\hline & $\begin{array}{l}20 \\
\mathrm{U} .\end{array}$ & $\begin{array}{l}10 \\
\mathrm{U} .\end{array}$ & $\begin{array}{l}2 \\
\mathrm{U}\end{array}$ & $\begin{array}{l}60 \\
\mathrm{meg} .\end{array}$ & $\begin{array}{c}30 \\
\text { meg. }\end{array}$ & $\begin{array}{c}10 \\
\mathrm{meg} .\end{array}$ & $\begin{array}{c}60 \\
\text { meg. }\end{array}$ & $\begin{array}{c}30 \\
\mathrm{meg}\end{array}$ & $\begin{array}{r}10 \\
\mathrm{meg} .\end{array}$ \\
\hline Streptococcus aga. & & & & & & & & & \\
\hline $\begin{array}{r}\text { lactiae } \ldots \ldots \\
\text { Streptococous } \\
\text { aga }\end{array}$ & 24,5 & 20 & 16,5 & 28 & 19,5 & 11,5 & 25 & 25 & 19,5 \\
\hline $\begin{array}{r}\text { lactiae } \ldots . . . \\
\text { Streptococcus aga- }\end{array}$ & 33 & 27 & 21,5 & 33,5 & 24 & 16,5 & 25 & 17 & 12,5 \\
\hline $\begin{array}{r}\text { lactiae } \\
\text { Streptococcus } \\
\text { Stys }\end{array}$ & 24 & 18 & 14,5 & 33,5 & 24 & 12,5 & 17 & 14 & 11,5 \\
\hline $\begin{array}{r}\text { galactiae } \\
\text { Streptococcus } \\
\text { ube. }\end{array}$ & 29 & 24 & 19,5 & 35 & 29,5 & 20 & 27 & 24 & 20,5 \\
\hline ris $\ldots . . .$. & 25 & 20 & 17 & 30,5 & 17 & $13,5^{\circ}$ & 30 & 27 & 22,5 \\
\hline Staphilococcus .. & 27 & 22 & 20,5 & 30 & 22 & 18 & 24 & 22,5 & 21 \\
\hline
\end{tabular}

Hospital " de New-York. L'agent producteur de cet antibiotique est un parasubtilis.

Les recherches effectuées par les découvreurs de la Bacitracine, ainsi que les magnifiques travaux de LEvaditi et Collaborateurs, ont permis d'établir son spectre d'activité antibactérienne vis-àvis de divers Streptococcus et Staphylococcus aureus, tout en signalant son action inhibitrice moins accentuée sur les germes des espèces Escherichia Coli et Pseudomonas.

En égard à sa toxicité, LEvaDITI considère que certains phénomènes toxiques observés avec cet antibiotique, sont dus à des impuretés associées au médicament, et spécialement à une substance histaminique.

Quant à l'utilité possible de cette médication dans le traitement des mammites bovines, Bensor (1948) déduit de ses expériences qu'à doses analogues à celles utilisées pour la Pénicilline (100.000 U. I.), la Bacitracine est aussi efficace que celle-là, opinant même que certains cas de mammites résistantes à la Pénicilline ne le sont pas à la Bacitracine.

Pour les considérations exposées ci-dessus, nous estimons d'intérêt l'emploi de cet antibiotique et, en même temps qu'à l'évaluation de son efficacité thérapeutique sur les bovins malades, il devra être procédé à une étude approfondie de son élimination par le lait et à la détermination de l'action de ce médicament sur les ferments des fromages élaborés dans les différents pays. 
L'état actuel du problème de la production d'un lait exempt de vestiges d'antibiotiques, ainsi que la nécessité de combattre les infections mammaires du bétail bovin, évitant par là les pertes f́levées qu'elles occasionnent, conseillent une collaboration loyale entre éleveurs, vétérinaires et industriels laitiers, en vue de mettre en pratique des mesures analogues à celles établies en Suède, et selon lesquelles le vétérinaire devra communiquer l'application d'antibiotiques aux laiteries qui excluront, en conséquence, le lait de toute transformation industrielle durant soixante-douze heures.

Le lait contenant de la Pénicilline pourra être utilisé pour l'alimentation des veaux, volailles, pores, etc.

L'obtention d'un lait pur, provenant de vaches cliniquement et bactériologiquement saines, constitue la tâche que nous, industriels, industriels, éleveurs et techniciens, sommes tenus de poursuivre et que nous poursuivons en collaborant à cette entreprise qui, par son extension et intérêt, portera bénéfice à la population terrestre entière.

\section{Conclusions}

1. Dans la fabrication des fromages espagnols, à base de lait de vache, type demi-dur léonais, des concentrations de 0,25-0,50 U. I. de Pénicilline par centimètre cube, ne rendent pas diffieile une fermentation normale. Des concentrations supérieures à $0,50 \mathrm{U}$. I. par centimètre cube, ont provoqué des modifications dans la morphologie et la vitalité des ferments lactiques, arrivant même à produire des troubles fermentatifs qui, altérant les caractéristiques organo-. leptiques des fromages élaborés, diminuent leur qualité.

2. On est arrivé à isoler, durant les différentes phases de la maturation, les espèces bactériennes qui interviennent dans ce processus biologique.

3. Aucune altération due à la flore de putréfaction, ni aucun gonflement produit par la croissance des espèces Escherichia Coli et Aerobacter aerogenes, n'ont été observés dans les produits fabriqués aveo du lait contenant $10 \mathrm{U}$. I. de Pénicilline par centimètre cube.

4. Les recherches effectuées au moyen du test des disques et l'emploi de diverses concentrations de six antibiotiqueśs différents, dans le but d'évaluer la sensibilité des ferments lactiques isolés, mettent en évidence (voir tableaux I et II) que la Bacitracine est la substance qui exerce l'action inibitrice la moins accentuée sur cette flore lactique qui participe de façon si active et fondamentale dans les processus biochimiques de la maturation du caillé employé dans la fabrication des fromages étudiés. 


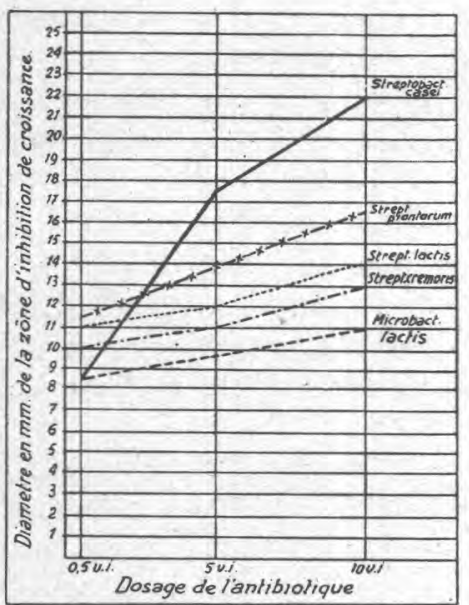

II. Etude comparative des zones d'inhíbition en $\mathrm{mm}$. de la eroissance des germes lactiques mentionnés, avec des concentrations de "Streptomycine" de 110. 10-1 meg., et disques de $6,5 \mathrm{~mm} / \mathrm{d}$.

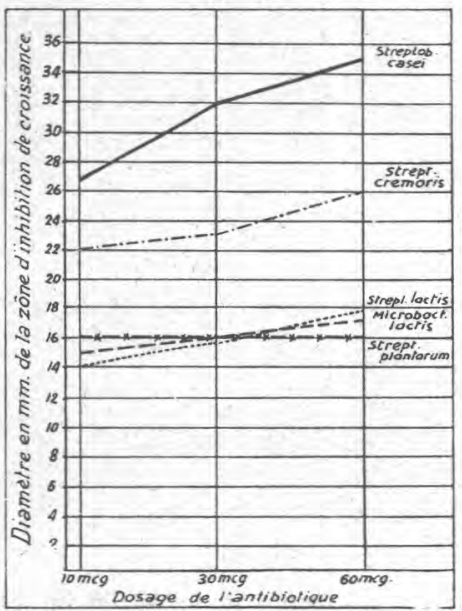

I. Etude comparative des zones d'inhibition en $\mathrm{mm}$. de la croissance des germes lactiques mentionnés, avec des concentrations de "Pénicilline * de 10-5-0,5 I. U., et disques de $6,5 \mathrm{~mm} / \mathrm{d}$.

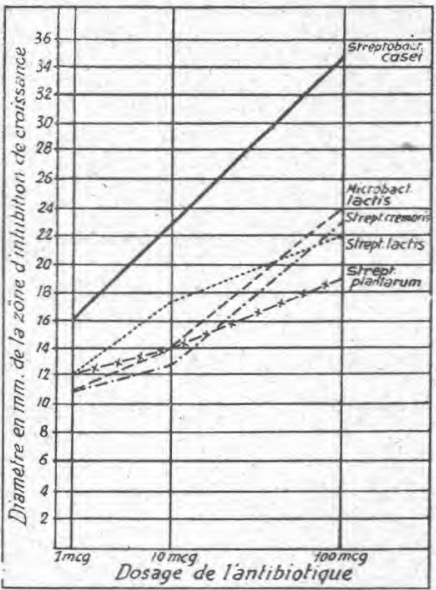

III. Etude comparative des zones d'inhibition en $\mathrm{mm}$. de la croissance des germes lactiques mentionnés, avec des concentrations d" "Auréomyeine " de 60-30-10 meg., et disques de $6,5 \mathrm{~mm} / \mathrm{d}$. 
IV. Etude comparative des zones d'inhibition en $\mathrm{mm}$. de la croissance des germes lactiques mentionnés, avec des concentrations de "Bacitracine" de 20 . $10.2 \mathrm{U}$., et disques de $6,5 \mathrm{~mm} / \mathrm{d}$.
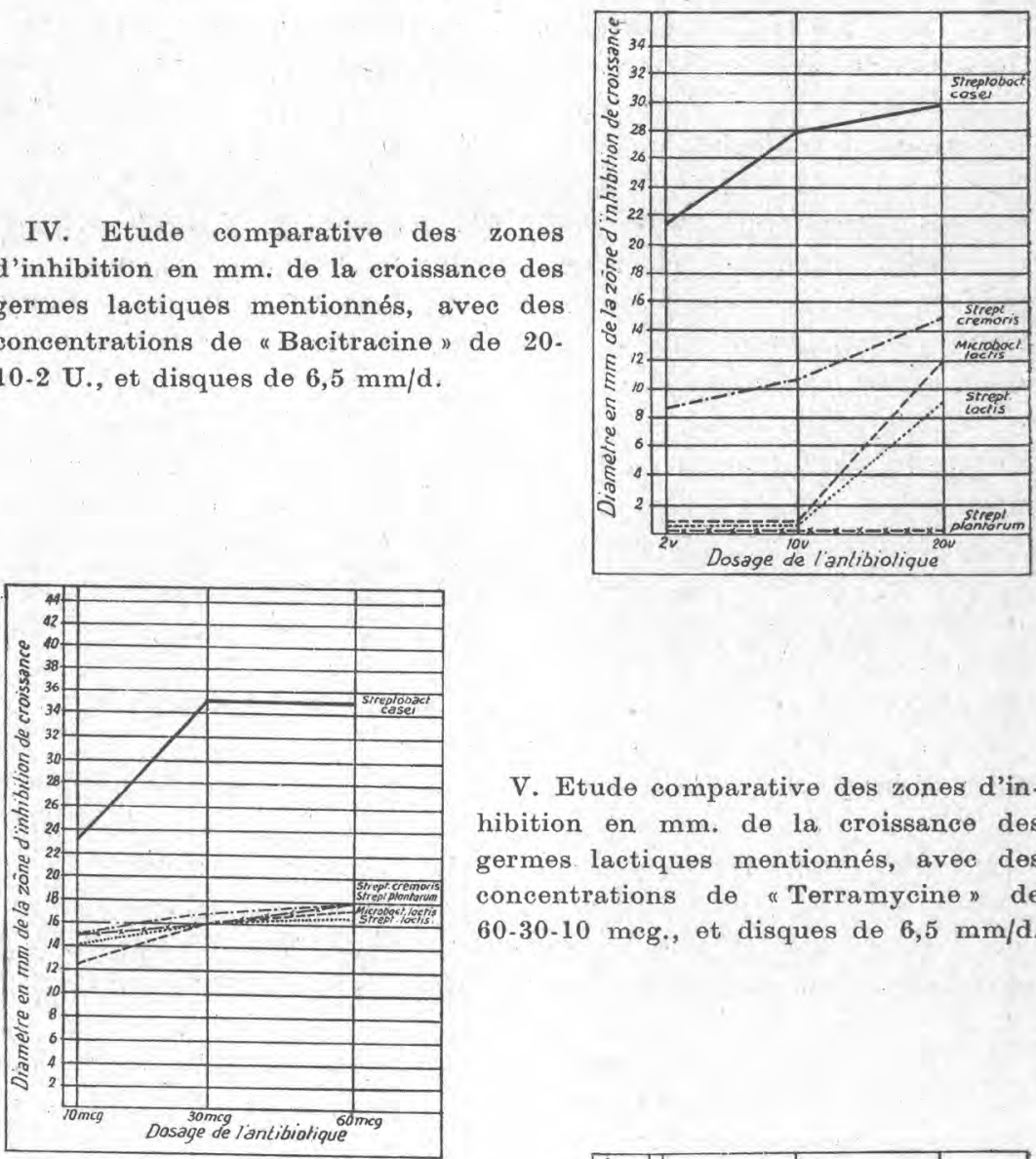

V. Etude comparative des zones d'inhibition en $\mathrm{mm}$. de la eroissance des germes lactiques mentionnés, avec des concentrations de "Terramycine" de 60-30-10 meg., et disques de 6,5 mm/d.

VI. Etude comparative des zones d'in. hibition en $\mathrm{mm}$. de la croissance des germes lactiques mentionnés, avec des concentrations de "Chloromycétine» de 60-30-10 meg., et disques de $6,5 \mathrm{~mm} / \mathrm{d}$.

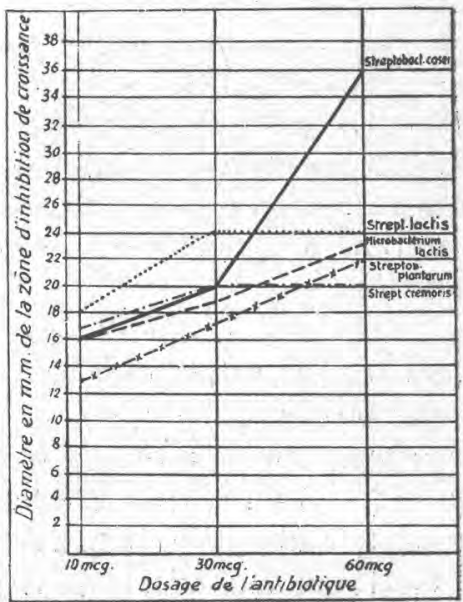


5. Les recherches réalisées en Espagne en vue de déterminer la flore des mammites latentes d'origine microbienne, nous ont fait connaître que $87 \%$ d'entre elles sont provoqués par les Streptococcus agalactiae, dysgalactiae et uberis et $13 \%$ seulement par les Staphylococcus, n'ayant jusqu'à présent identifié aucun agent bactérien des espèces Escherichia on Corynebacterium. Toutefois, nous estimons que ces derniers germes interviennent également, quoiqu'en proportion réduite, dans l'étiologie des processus mammaires bactériens de caractère infecto-contagieux, et uniquement, en outre, dans certaines formes cliniques de l'infection.

6. Durant l'étude réalisée pour l'évaluation, au moyen de l'épreuve des disques, dc la sensibilité de la flore pathogène des mammites, vis-à-vis des antibiotiques, nous avons pu constater (voir tableaux III et IV) que la Bacitracine exerce une action inhibitrice intense sur les Streptococcus et Staphylococcus isolés d'infections mammaires, motif pour lequel nous considérons convenable de continuer l'essai thérapeutique de cet antibiotique (Bacitracine), jusqu'à ce qu'on ait trouvé une thérapeutique qui respecte la flore microbienne normale du lait et des eaillés utilisés dans la fabrication des fromages.

7. Considérant que le lait, tant pour sa consommation à l'état frais que pour son industrialisation, ne doit contenir aucune substance étrangère (antibiotiques, pénicillinase, hydroxylamine, etc.), il est proposé que la constatation de l'une quelconque de ces substances soit considérée comme une fraude aux fins d'application de sanctions.

Il est également proposé de conseiller aux Gouvernements respectifs la rédaction de dispositions dans le sens indiqué au paragraphe antérieur.

8. En vue d'obtenir la collaboration entre éleveurs et industriels, il est recommandé que les Comités Nationaux accomplissent, au moyen de la radio, de conférences, etc., une vaste tâche éducatrice des éleveurs, afin d'empêcher, entre autres, la remise, à l'industrie, de laits contenant de la Pénicilline ou autres antibiotiques durant les trois jours suivant la dernière application du médicament.

9. Dans le but d'éviter des perturbations dans les industries du lait, il devra être sollicité des Gouvernements respectifs que l'application d'antibiotiques aux animaux laitiers soit toujours réalisée sous la direction et surveillance vétérinaires.

10. Il y a intérêt à continuer les recherches tendant à la découverte d'une méthode dévoilant rapidement la présence d'anti biotiques dans le lait de mélange. 


\title{
BIBLIOGRAPHIE
}

Orla-Jensen. The lactic acid bacteria, 1919.

BERGEY. Manual of determinative bacteriology, 1948.

A. W. Stableforth. Bull. Office Int. Epizooties, XXX, 121, 1948.

P. Kästrı. Bull. Office Int. Epizooties, XXX, 144, 1948.

H. F. Farrag. Jour. Amer. Vet. Med. Assoc., 371, 1948.

Benson. Jour. Amer. Vet. Med. Assoc., 1948. Ciencia Veterinaria, 1949.

J. P. Marty. La Pénicilline, Vigot Frères, Paris, 1948.

H. KatruchoN et E. G. Hodd. Journal Dairy Science, XXXII, 1949.

Levaditi. Antibiotiques, I. B. Baillière, Paris, 1950.

Aage Jepsen et A. J. Overby. The Nether milk and Dairy Jour., 5, 1951.

R. N. Gostilow et M. L. Speck. Jour. of Dairy Science, XXXIV, 34, 1951.

L. E. Baribo et E. M. Foster. Jour. of Dairy Science, XXXIV, 34, 1951.

Santos Ovejero. Veterinaria, 1951.

S. C. Bryan. Jour, Milk Food Techn., 14, 161, 1951.

W. A. Krienke. Milk Dealer, 104, 1951.

Legislacion (SUecta). Extracto de Le Lait, 307, 1951.

J. Auclatr. Le Lait, XXXI, 121, 1951.

J. Jacquet et P. Julienne. Bull. Acad. Vet., 24, 423, 1951.

K. Е. Тноме́. F.I.L., 1952.

A. Bradfield, L. A. Resi et D. B. Johnstone. Jour. Dairy Science, XXXV, 1952.

R. Willems. Bull. Office Int. Epizooties, XXXVIII, 390, 1952.

Kästl. Extrac. Le Lait, XXXII, 554, 1952.

J. Jacquet, R. Richou, Mc Steeg, C. Gerbaux et P. Julienne. $C$. R. Acad. Sc., 1952.

Santos Ovejero. Rev. Espanola de Lecheria, 5, 273, 1952.

M. M. Lemoine, G. Saucher et H. Girard. Le Lait, XXXIII, 15, 1953.

\section{ESSAIS DE MISE EN CONSERVE DE LAIT ÉCRÉMÉ PAR ENSILAGE (1)}

\author{
par
}

\section{DESOUTTER}

Dans le rapport que j'ai eu l'honneur de vous présenter l'an dernier sur la production laitière en France, j'indiquais que cette production était en augmentation constante, alors que les débouchés étrangers se fermaient à nous, en raison des charges multiples qui pèsent sur nos prix de revient et ne nous permettent pas de lutter à armes égales avec les nations qui nous entourent; et j'ajoutais que la fièvre aphteuse pourrait peut-être ajourner quelque peu une crise vers laquelle nous nous acheminons, mais ne résoudrait pas le problème.

(1) Comptes Rendus de l'Académie Agric., 1953, 39, n० 7, 385. 